\title{
Beta 2-Band Synchronization during a Visual Oddball Task
}

\author{
M. KUKLETA ${ }^{1,3}$, P. BOB ${ }^{1,3}$, M. BRÁZDIL ${ }^{2}$, R. ROMAN ${ }^{1}$, I. REKTOR ${ }^{2}$ \\ ${ }^{1}$ Department of Physiology and ${ }^{2}$ First Department of Neurology, Medical Faculty, Masaryk \\ University, Brno, ${ }^{3}$ Department of Psychiatry, First Medical Faculty, Charles University, Prague, \\ Czech Republic
}

Received June 17, 2008

Accepted September 5, 2008

On-line November 4, 2008

\section{Summary}

The study investigated whether specific changes in phase synchrony in the beta 2 frequency band of EEG $(25-35 \mathrm{~Hz})$ occurred during a recognition task. The level of synchrony was examined between one hundred and eighty loci in the frontal and temporal lobes of eight epileptic patients with intracerebral electrodes; the EEG records were obtained during a visual oddball task. In each pair of records, the correlation curves were created from the sequence of correlation coefficients calculated. These curves consisted of irregular oscillations between the maximal and minimal $r$-values. Transient highly synchronized activity was observed during the whole time course of the experiment in all record pairs investigated and a significant relationship was found between the number of such episodes and the mean correlation coefficient (Spearman R 0.84; N 3240; $\mathrm{p}<0.001)$. On averaged curves, which were calculated using stimulus onsets as the trigger of averaging, a significant increase of the mean correlation coefficient in the post-stimulus epoch was found $(p<0.01$ after both target and non-target stimuli; $t$-test for dependent samples). As the cognitive demand significantly increases after stimulus presentation, the results are considered to be the first evidence from intracranial recording of increased synchronization in the beta 2 frequency band related to the cognitive activity.

\section{Key words}

Intra-cerebral EEG recording in humans • Visual oddball task • Beta 2 synchronization • Binding in cognition

\section{Corresponding author}

M. Kukleta, Department of Physiology, Komenskeho 2, 66243 Brno, Czech Republic. Tel.: 00420549495 117. E-mail: mkukleta@med.muni.cz

\section{Introduction}

The accumulating evidence from experimental studies has demonstrated the relationship of synchronization of neuronal activity to several complex brain functions. Functionally relevant epochs of gammaband synchronization have been observed in various species and brain structures during attention, perception, motor and memory tasks (Lee et al. 2003). The direct link between visual perception and gamma synchrony was demonstrated in the cat visual cortex (Eckhorn et al. 1988, Gray et al. 1989). Following this finding, functional significance of synchronous gamma activity in selective attention, perceptual processing and recognition was repeatedly demonstrated in animal and human studies (Lutzenberger et al. 1995, Muller et al. 1997, Rodrigez et al. 1999, 2004, Klemm et al. 2000, Fries et al. 2001, Bhattacharya et al. 2001). In the case of sensory awareness, synchronous activity was suggested to generate coherent internal states and to achieve perceptual selection that could be crucial for controlling the access of information to consciousness (Menon et al. 1996, Herrmann et al. 1999, Engel and Singer 2001, Meador et al. 2005). The significance of synchronized gamma oscillations was also demonstrated in somatomotor activity (Baker et al. 2001, Ohara et al. 2001, Schofellen et al. 2005). As a whole these findings indicate that selective neuronal inter-regional synchronization may underlie the binding of multiple and disparate neural activities into the functional whole (Bressler et al. 1993, Lee et al. 2003). It is worth noticing that increased gamma coherence was observed frequently among diverse regions of both hemispheres, suggesting 
that binding is subserved by high frequency synchronization among widespread brain networks.

In the present study, we have investigated synchronization of EEG activity during a visual oddball task performed by epileptic patients with intracerebral electrodes, which were implanted before a surgical treatment. The study character was explorative. Our data made it possible to assess synchronization of local field potentials between frontal and temporal recording sites in a representative number of cases (180 correlated pairs). In contrast to the majority of studies aimed to gamma band synchronous activity, we have focused to beta 2 band (25-35 Hz) with the purpose to examine whether specific changes in synchronous activity also occur in this band.

\section{Methods}

\section{Subjects}

Eight patients (5 males, 3 females; aged 25-45 years; mean 32.4 years; all with medically intractable epilepsies; all right handed) participated in the study. Standard MicroDeep semi-flexible sheaves of electrodes (DIXI) with the diameter of $0.8 \mathrm{~mm}$, length of each electrode $2 \mathrm{~mm}$, and inter-electrode intervals of $1.5 \mathrm{~mm}$ were used for invasive EEG monitoring. The orthogonal depth sheaves were implanted in the frontal, temporal, and/or parietal lobes using the methodology by Talairach et al. (1967) with the aim to localize the seizure origin prior to a surgical treatment. In two patients, additional diagonal sheaves of electrodes were inserted stereotactically into the amygdalo-hippocampal complex (via frontal approach, passing through the basal ganglia in one patient, via occipital approach in the other). The sheaves of electrodes were placed bilaterally in six patients and unilaterally in two patients. Electrodes at the sheaf (5-15) were always numbered from the medial to lateral sites. Their positions were indicated in relation to the axes defined by Talairach system (1967) using the ' $x, y, z$ ' format where ' $\mathrm{x}$ ' is lateral, millimeters to midline, positive right hemisphere, ' $y$ ' is antero-posterior, millimeters to the AC (anterior commissure) line, positive anterior, and ' $\mathrm{z}$ ' is vertical, millimeters to the $\mathrm{AC} / \mathrm{PC}$ (posterior commissure) line, positive up. The exact positions of sheaves and their electrodes in the brain were verified using post-placement magnetic resonance imaging with electrodes in situ. The recordings from lesional structures and epileptogenic zones were not included into the analysis. No patient from the group examined has had bilateral hippocampal sclerosis or bilateral temporal lobe epilepsy. All the patients had normal or corrected-to-normal vision. Informed consent was obtained from each patient prior to the experiment, and the study received an approval from the Ethical Committee of Masaryk University.

\section{Procedure}

The patients were seated comfortably in a moderately lighted room with a monitor screen positioned approximately $100 \mathrm{~cm}$ in front of their eyes. During the examination they were asked to focus the gaze continuously on the point in the center of the monitor screen and to respond, as quickly as possible, to a target stimulus (yellow letter $\mathrm{X}$ on the white background) by pressing a micro-switch button in the dominant hand and counting the number of these stimuli in their heads, and to ignore frequent stimuli (yellow letter $\mathrm{O}$ on the white background). Both stimuli were displayed on the black screen, subtended at the visual angle of $3^{\circ}$. Their duration was $200 \mathrm{~ms}$. The inter-stimulus intervals varied randomly between 2 and $5 \mathrm{~s}$, the ratio of target to frequent, nontarget stimuli was 1:5. Mean duration of the whole experiment was $17.4 \mathrm{~min}$ (minimum $12.8 \mathrm{~min}$, maximum $20.7 \mathrm{~min})$.

\section{EEG recording}

The EEG signal was recorded simultaneously from various intracerebral structures using 64 channel Brain Quick EEG system (Micromed). The recordings were monopolar with respect to a reference electrode placed on the right processus mastoideus in all the cases. EEGs were amplified with the bandwidth of 0.1-40 Hz at the sampling rate of $128 \mathrm{~Hz}$. ScopeWin software was used for the signal analysis, which included up to 44 channels recorded simultaneously.

\section{Data analysis}

The data for analysis of beta 2 synchronous oscillations in remote brain areas were obtained from 180 pairs of local field potential records. 106 pairs were homolateral (70 pairs in the left, 36 pairs in the right hemisphere), 74 pairs were heterolateral. All the available pairs of frontal and temporal records were analyzed. The detailed information about location of recording electrodes, from which investigated records were derived, is given in Table1. All the selected recording sites exhibited an evoked response to target and non-target stimuli. 
Table 1. Location of recording electrodes, which represented the investigated pairs.

\begin{tabular}{|c|c|c|c|}
\hline \multicolumn{2}{|c|}{ Location of recording electrode } & \multirow{2}{*}{$\begin{array}{c}\text { Number of patients } \\
6\end{array}$} & \multirow{2}{*}{$\begin{array}{c}\text { Number of pairs } \\
22\end{array}$} \\
\hline G cinguli & G temp sup, med, inf & & \\
\hline G cinguli & G fusiformis & 2 & 2 \\
\hline G cinguli & G parahippocamp & 4 & 4 \\
\hline G cinguli & Hippocampus & 5 & 11 \\
\hline G cinguli & Amygdala & 5 & 6 \\
\hline$G$ orbitales & G temp sup, med, inf & 6 & 28 \\
\hline G orbitales & G fusiformis & 2 & 3 \\
\hline G orbitales & G parahippocamp & 3 & 6 \\
\hline G orbitales & Hippocampus & 4 & 9 \\
\hline G orbitales & Amygdala & 7 & 10 \\
\hline$D L P F C$ & G temp sup, med, inf & 2 & 8 \\
\hline$D L P F C$ & G fusiformis & 2 & 2 \\
\hline$D L P F C$ & $G$ parahippocamp & 1 & 1 \\
\hline$D L P F C$ & Hippocampus & 2 & 2 \\
\hline$D L P F C$ & Amygdala & 3 & 3 \\
\hline Motor cortices & G temp sup, med, inf & 1 & 5 \\
\hline Motor cortices & $G$ parahippocamp & 1 & 3 \\
\hline Motor cortices & Amygdala & 1 & 1 \\
\hline G rectus & G temp sup, med, inf & 2 & 7 \\
\hline$G$ rectus & G parahippocamp & 1 & 1 \\
\hline$G$ rectus & Hippocampus & 2 & 4 \\
\hline$G$ rectus & Amygdala & 2 & 3 \\
\hline$G$ front med & G temp sup, med, inf & 4 & 14 \\
\hline$G$ front med & G fusiformis & 1 & 1 \\
\hline$G$ front med & G parahippocamp & 1 & 1 \\
\hline$G$ front med & Hippocampus & 3 & 8 \\
\hline$G$ front med & Amygdala & 4 & 7 \\
\hline$G$ front inf & G temp sup, med, inf & 1 & 3 \\
\hline$G$ front inf & Hippocampus & 1 & 3 \\
\hline$G$ front inf & Amygdala & 1 & 2 \\
\hline
\end{tabular}

G - gyrus, gyri; DLPFC - dorsolateral prefrontal cortex; G front med - gyrus frontalis medialis (20) and medius (11); G front inf - gyrus frontalis inferior.

The processing of records comprised their frequency decomposition and computation of the phase correlation in pairs of these records. At first, the wholeband EEG record from the whole experiment was reduced to beta 2 frequency band $(25-35 \mathrm{~Hz})$ via digital bandpass filter. The procedure comprised the spectrum computation using Fast Fourier Transformation, zeroing all the spectral components outside the selected frequency interval, and inverse complex Fast Fourier Transformation computation. Then the correlation coefficients were calculated in pairs of these filtered records using the technique of running correlation. The length of sliding window in the computation of successive correlation coefficients was 12 points (94 ms). ScopeWin software was used for the data processing.

Two methodical approaches were used in analyzing the correlation curves obtained by the procedure described. In the first one, the averaged curves were calculated using stimulus onset as the trigger and analyzed (averaged data analysis). Mean number of averaged stimuli was 48 (minimum 36, maximum 61) in the case of target stimuli, and 136 (minimum 90, maximum 165) in the case of non-target stimuli. In the second approach, eighteen 30-s segments covering the 
whole experiment were analyzed (raw data analysis). In both cases, the artifact-free EEG segments were included into analysis only (selection was based on the visual inspection of the segments by an experienced person).

Statistics were obtained by using the routines included in the program package Statistica '99 (StatSoft Inc, Tulsa, USA).

\section{Results}

The analysis of error responses to both nontarget and target stimuli was taken as the demonstration that patients really processed the stimuli according to the experimental instructions. Median percentage of errors was 1.3 (minimum 0 , maximum 4.8). Considering the total number of stimuli presented (median 320, minimum 262, maximum 397), this result was taken as sufficient evidence of patient's active participation in the experiment.

The sequence of correlation coefficients, which was obtained by running correlation technique formed curves illustrating the activity synchronization in pairs of selected remote brain sites (Fig. 1). These correlation curves consisted of irregular oscillations between the maximal and minimal $r$-values. The minimal peaks were more variable and frequently attained values of negative correlation. In this study, peaks with $r$ value of +0.9 and higher were used as indicators of high activity synchronization between examined loci; they were termed highly correlated or he segments (the choice of the value was arbitrary). Mean correlation coefficients, which were calculated in selected segments of correlation curves $(1 \mathrm{~s}$ before and $1 \mathrm{~s}$ after the stimulus presentation in averaged data analysis; in consecutive $30 \mathrm{~s}$ segments covering the whole experiment in raw data analysis), were the second indicator used in the study. These indicators were analyzed both in a composite of 180 readings of eight patients (merged data) and in singular patients (individual data).

The impact of increased cognitive activity on beta 2 synchronization was investigated on averaged correlation curves by comparing the pre-stimulus epoch (taken as a control condition) with post-stimulus epoch, during which the discrimination of stimuli, selection of an appropriate response and, in the case of target stimuli, its execution occurred. As evident from the data presented in Table 2, section A, the hc segments were observed in both the pre-stimulus and post-stimulus epochs ( $p$-value of the difference $>0.05$ in both non-target
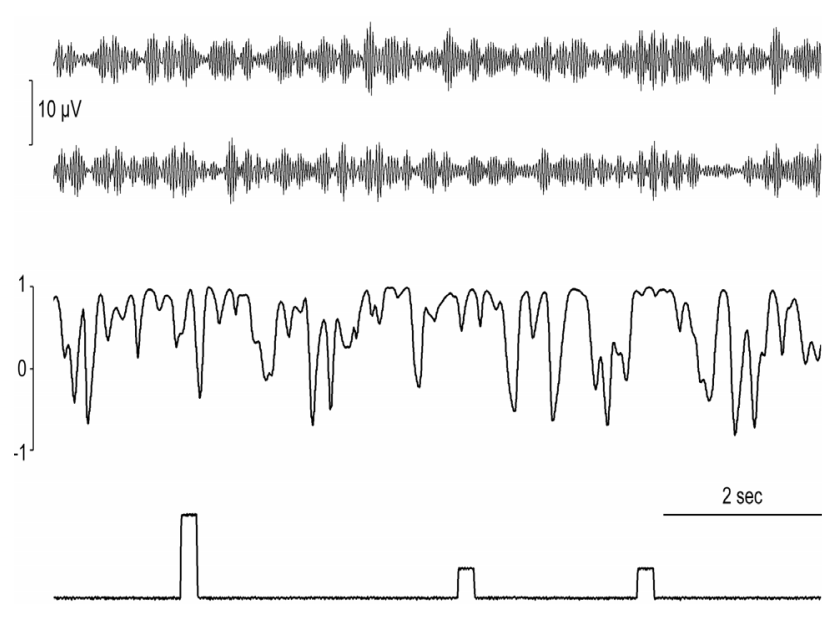

Fig 1. Upper two curves represent the beta 2 frequency components $(25-35 \mathrm{~Hz})$ of a segment of EEG records from the right dorsolateral prefrontal cortex and the right fusiform gyrus of patient No 2. Bottom two curves represent the correlation coefficients ( $r$ values from +1 to -1 ) calculated from the above pair of filtered records, and the position of non-target (smaller marks) and target (higher mark) stimuli on the time axis of the segment.

and target stimuli; Wilcoxon matched pairs test). Section B presents the comparison of mean correlation coefficients between pre- and post-stimulus periods. In contrast to the number of he segments, this indicator increased in post-stimulus epoch (t-values -2.8 in the case of non-target stimuli and -4.0 in the case of target stimuli; $\mathrm{p}<0.01$ in both cases; $t$-test for dependent samples). The same comparison in singular patients demonstrated significant post-stimulus increase of mean correlation coefficient in patients 2,4 , and 8 in the case of non-target stimuli (t-values $-2.9,-3.6$, and -3.8 , respectively; $\mathrm{p}<0.01$ in all cases; $t$-test for dependent samples) and in patients 1,2 , and 7 in the case of target stimuli (t-values $-3.2,-3.4$, and -8.6, respectively; $\mathrm{p}<0.01$ in all cases; $t$-test for dependent samples). All the other differences were not significant. In raw (non-averaged) data, hc segments were found in all the investigated pairs of each patient. They were observed throughout the experiment; no tendency to change in their incidence was observed. Table 3 presents mean numbers of hc segments calculated in eighteen 30 -s epochs of correlation curves. These results demonstrated a few facts:

a) There were significant inter-individual differences in the incidence of hc segments $(\mathrm{p}<0.01$ between patients 1-2, 1-4, 2-3, 2-5, 2-6, 2-7, 2-8, 3-4, 3-7, 4-5, 4-6, 4-7, 4-8, 5-7, 6-7; $\mathrm{p}<0.05$ between patient 1-7; one-way ANOVA, $\mathrm{N}=3240, \mathrm{~F}=174.7$ ).

b) In all the investigated pairs, the number of he segments in 30-s epochs varied greatly during the course 
Table 2. Number of highly correlated segments (A) and mean correlation coefficient (B) in 1 second pre-stimulus and post-stimulus periods of averaged correlation curves, which were created from 180 record pairs from 8 patients.

A

\begin{tabular}{lll}
$\begin{array}{l}\text { Averaged } \\
\text { Trials }\end{array}$ & $\begin{array}{l}\text { Median (min-max) of he segments } \\
(\mathbf{1} \text { s pre-stimulus period) }\end{array}$ & $\begin{array}{l}\text { Median (min-max) of he segments } \\
\text { (1 s post-stimulus period) }\end{array}$ \\
\hline $\begin{array}{l}\text { Non-target } \\
\text { Target }\end{array}$ & $1(0-4)$ & $\begin{array}{l}1(0-4) \\
1(0-4)\end{array}$ \\
\hline
\end{tabular}

$\underline{B}$

\begin{tabular}{lll}
$\begin{array}{l}\text { Averaged } \\
\text { Trials }\end{array}$ & $\begin{array}{l}\text { Mean value of correlation coefficient } \pm \text { SD } \\
\text { (1 s pre-stimulus period) }\end{array}$ & $\begin{array}{l}\text { Mean value of correlation coefficient } \pm \text { SD } \\
\text { (1 s post-stimulus period) }\end{array}$ \\
\hline $\begin{array}{l}\text { Non-target } \\
\text { Target }\end{array}$ & $0.19 \pm 0.24$ & $0.25 \pm 0.24$ \\
$0.15 \pm 0.27$ & $0.23 \pm 0.28$ \\
\hline
\end{tabular}

Table 3. Mean number of highly correlated segments ( $r$ value higher than 0.9 ) and its standard deviation calculated in eighteen 30-s segments of correlation curves.

\begin{tabular}{lllll}
\hline Patient & No of pairs & $\begin{array}{l}\text { Mean number of hc } \\
\text { segments (all pairs) }\end{array}$ & $\begin{array}{l}\text { Minimal mean } \\
\text { (1 pair) }\end{array}$ & $\begin{array}{l}\text { Maximal mean } \\
\text { (1 pair) }\end{array}$ \\
\hline 1 & 12 & $13.2 \pm 4.9$ & $8.3 \pm 3.0$ & $19.6 \pm 4.0$ \\
2 & 20 & $25.1 \pm 14.6$ & $8.7 \pm 3.3$ & $52.9 \pm 9.1$ \\
3 & 24 & $11.7 \pm 3.3$ & $9.6 \pm 2.7$ & $13.9 \pm 3.0$ \\
4 & 27 & $24.7 \pm 13.4$ & $9.4 \pm 2.5$ & $53.9 \pm 10.0$ \\
5 & 21 & $12.7 \pm 4.2$ & $8.8 \pm 2.9$ & $17.9 \pm 3.9$ \\
6 & 16 & $13.0 \pm 4.7$ & $8.9 \pm 4.0$ & $20.9 \pm 5.4$ \\
7 & 48 & $15.7 \pm 5.2$ & $10.8 \pm 3.2$ & $24.3 \pm 4.8$ \\
8 & 12 & $14.4 \pm 5.2$ & $9.4 \pm 2.9$ & $22.4 \pm 4.3$ \\
\hline
\end{tabular}

of experiment (see standard deviations of all presented means).

c) In all the patients, the mean number of hc segments varied according to the location of investigated pair (see, for instance, the differences between the maximal and minimal means).

The mean correlation coefficients calculated in eighteen 30-s epochs of correlation curves are presented in Table 4. As evident, this indicator exhibited very similar characteristics as he segments:

a) There were significant inter-individual differences in the mean value of correlation coefficients ( $\mathrm{p}<0.01$ between patients 1-2, 1-4, 1-7, 2-3, 2-5, 2-6, 2-7, 2-8, 3-4, 3-7, 3-8, 4-5, 4-6, 4-7, 4-8, 5-7, 5-8, 6-7, 6-8; one-way ANOVA, $\mathrm{N}=3240, \mathrm{~F}=235.8$ ).

b) In all the investigated pairs, the mean value of correlation coefficient in 30-s epochs varied greatly during the course of experiment (see standard deviations of all presented means).

c) In all the patients, the mean value of correlation coefficient in 30 -s epochs varied greatly according to the location of investigated pair (see, for instance, the differences between maximal and minimal means).

The calculation of correlation between the number of hc segments and mean value of correlation coefficients answered the question about possible relationship of these two indicators. Spearman $R$ coefficient calculated from the data of all pairs of all patients $(\mathrm{N}=3240)$, was $0.84(0.83$ in patient $1,0.95$ in patient $2,0.55$ in patient $3,0.95$ in patient $4,0.73$ in patient $5,0.70$ in patient $6,0.77$ in patient 7 , and 0.79 in 
Table 4. Mean value of correlation coefficient and its standard deviation calculated in eighteen 30-s segments of correlation curves.

\begin{tabular}{lllll}
\hline Patient & No of pairs & $\begin{array}{l}\text { Mean r-value } \\
\text { (all pairs) }\end{array}$ & $\begin{array}{l}\text { Minimal r-value } \\
\text { (1 pair) }\end{array}$ & $\begin{array}{l}\text { Maximal r-value } \\
\text { (1 pair) }\end{array}$ \\
\hline 1 & 12 & $0.13 \pm 0.09$ & $0.02 \pm 0.04$ & $0.25 \pm 0.03$ \\
2 & 20 & $0.30 \pm 0.17$ & $0.06 \pm 0.04$ & $0.58 \pm 0.05$ \\
3 & 24 & $0.10 \pm 0.05$ & $0.05 \pm 0.03$ & $0.14 \pm 0.04$ \\
4 & 27 & $0.29 \pm 0.17$ & $0.04 \pm 0.03$ & $0.60 \pm 0.06$ \\
5 & 21 & $0.10 \pm 0.07$ & $0.01 \pm 0.03$ & $0.18 \pm 0.04$ \\
6 & 16 & $0.10 \pm 0.07$ & $0.02 \pm 0.05$ & $0.24 \pm 0.06$ \\
7 & 48 & $0.17 \pm 0.08$ & $0.08 \pm 0.04$ & $0.31 \pm 0.03$ \\
8 & 12 & $0.15 \pm 0.12$ & $0.01 \pm 0.04$ & $0.29 \pm 0.06$ \\
\hline
\end{tabular}

patient 8$)$. All these correlations were significant $(\mathrm{p}<0.01$ in all cases).

\section{Discussion}

The increase of mean r-values in post-stimulus period of averaged curves from both the non-target and target trials belongs to the main results of the study. Together with demonstrated correlation of this indicator with the number of he segments ( $\mathrm{R}=0.84)$, this finding suggests that beta 2 synchrony relates, at least in some cases, to cognitive function. The transient highly synchronized beta 2 activity observed between remote brain sites during the whole time course of experiment in all of 180 pairs of recording sites was another important finding. The fact that both mean r-values and hc segments have shown large variability that was related to the individual, localization of recording electrodes, and time course of experiment opens interesting directions for further research in this field.

On theoretical grounds, the transient and context-dependent precise temporal synchrony in neuronal firing represents a serious candidate for binding mechanism. It would selectively tag the responses of neurons that code for one event and demarcate their responses from those of neurons activated by other event. This highly selective temporal structure would allow the co-activation of multiple assemblies in the same network, which nonetheless remain distinguishable. The temporal binding could also serve as a mechanism for the selection of assemblies for further processing, because precisely synchronized neurons constitute salient state, which can be detected by coincidence-sensitive neurons in other brain areas (Abeles 1982, Konig et al. 1996). These neurons could in turn become organized into other assemblies which assure further processing of selected activation patterns. From this point of view, the elevation of beta 2 synchrony in post-stimulus epochs (evidenced indirectly by an increase of mean $r$-values and their linkage to the number of hc segments) could be considered as a manifestation of binding activity. This interpretation is in agreement with the reported involvement of gamma EEG synchronization in cognitive activity, although our data does not allow to discuss in more details the relevance of these two findings.

Recent data have shown that widespread and robust gamma activation of cortical EEG occur during the expectancy, learning, reading, and subtraction tasks (Engel and Singer 2001, Fitzgibbon et al. 2004, Meador et al. 2005). The increasing evidence also indicate that gamma band response can be reliably elicited during simple object recognition paradigms as a response to various types of stimuli such as faces, words and familiar line drawings (Pulvermuller 1996, Rodriguez et al. 1999, Gruber et al. 2002, Fiebach et al. 2005, Gruber and Muller 2005, Gruber et al. 2006, Freunberger et al. 2007) and may represent a correlate with the activation of distinct cortical representations of processed sensory object (Tallon-Baudry and Bertrand 1999). As the reported beta 2 band synchronization during recognition of target and non-target stimuli were not statistically different, the results of this study are also in agreement with the opinion that gamma band synchronization does not allow to distinguish among several types of meaningful stimuli (Freunberger et al. 2007).

The results have also shown variability of mean $r$-value in different segments of the correlation curve that was typically specific for each individual subject, pair of recording sites, and time course of experiment. Such kind of dependency suggests a presence of specific factors that 
might characterize interregional relationships of the neuronal network and specific individual features of cognitive style. Time variability and fluctuations of interregional correlations also demonstrate that the brain web represents the dynamic network of interregional connections.

Finally, the inconsistency concerning the behavior of two indicators investigated in average and raw data analyses should be discussed. Although the mean number of hc segments and the mean correlation coefficient were highly correlated and exhibited similar characteristics in raw data analysis, they failed to demonstrate the same when average data were analyzed. The limited number of values from which the average of the first indicator was calculated (approximately a hundredfold lower number as compared with the second indicator) was very probably the reason for its low sensitivity in this case.

In summary, although results of this study are in principle in line with recent findings, they have represented the first result from intracranial recording that reports the increased synchronization in beta 2 frequency band during a recognition task. The demonstrated linkage of binding specific processes and individual characteristics presents interesting perspective for further research. It could implicate new findings for better understanding of specific cognitive style and/or pathology as related to binding mechanism and specific integrative processes reflecting fundamental selforganization within the brain.

\section{Conflict of Interest}

There is no conflict of interest.

\section{Acknowledgements}

Authors thank for support by grants MSM 0021620849 and MSM 0021622404.

\section{References}

ABELES M: Role of the cortical neuron: integrator or coincidence detector? Isr J Med Sci 18: 83-92, 1982.

BAKER SN, SPINKS R, JACKSON A, LEMON RN: Synchronization in monkey motor cortex during a precision grip task. I. Task dependent modulation in single-unit synchrony. J Neurophysiol 85: 869-885, 2001.

BHATTCHARYA J, PETSCHE H, PEREDA E: Long-range synchrony in the gamma band: role in music perception. J Neurosci 21: 6329-6337, 2001.

BRESSLER SL, COPPOLA R, NAKAMURA R: Episodic multiregional cortical coherence at multiple frequencies during visual task performance. Nature 366: 153-156, 1993.

ECKHORN R, BAUER R, JORDAN W, BROSCH M, KRUSE W, MUNK M, REITBOECK HJ: Coherent oscillations: A mechanism of feature linking in the visual cortex? Multiple electrode and correlation analysis in the cat. Biol Cybern 60: 121-130, 1988.

ENGEL AK, SINGER W: Temporal binding and the neural correlates of sensory awareness. Trends Cognitive Sci 5: 16-25, 2001.

FIEBACH CJ, GRUBER T, SUPP G: Neuronal mechanisms of repetition priming in occipitotemporal cortex: spatiotemporal evidence from functional magnetic resonance imaging and electroencephalography. J Neurosci 25: 3414-3422, 2005.

FITZGIBBON SP, POPE KJ, MACKENZIE L, CLARK CR, WILLOUGHBY JO: Cognitive tasks augment gamma EEG power. Clin Neurophysiol 115: 1802-1809, 2004.

FREUNBERGER R, KLIMESCH W, SAUSENG P, GRIESMAYR B, HOLLER Y, PECHERSTORFER T, HANSLMAYR S: Gamma oscillatory activity in a visual discrimination task. Brain Res Bull 71: 593-600, 2007.

FRIES P, REYNOLDS JH, RORIE AE, DESIMONE R: Modulation of oscillatory neuronal synchronization by selective visual attention. Science 291: 1560-1563, 2001.

GRAY CM, KONIG P, ENGEL AK, SINGER W: Oscillatory responses in cat visual cortex exhibit inter-columnar synchronization which reflects global stimulus properties. Nature 338: 334-337, 1989.

GRUBER T, KEIL A, MULLER MM: Modulation of induced gamma band responses in a perceptual learning task in human EEG. J Cogn Neurosci 14: 732-744, 2002. 
GRUBER T, MULLER MM: Oscillatory brain activity dissociates between associative stimulus content in a repetition priming task in the human EEG. Cereb Cortex 15: 109-116, 2005.

GRUBER T, TRUJILLO-BARRETO NJ, GIABBICONI CA, VALDES-SOSA PA, MULLER MM: Brain electrical tomography (BET) analysis of induced gamma band responses during a simple object recognition task. Neuroimage 29: 888-900, 2006.

HERRMANN CS, MECKLINGER A, PFEIFER E: Gamma responses and ERPs in a visual classification task. Clin Neurophysiol 110: 636-642, 1999.

KLEMM WR, LI TH, HERNANDEZ JL: Coherent EEG indicators of cognitive binding during ambiguous figure tasks. Conscious Cogn 9: 66-85, 2000.

KONIG P, ENGEL AK, SINGER W: Relation between oscillatory activity and log-range synchronization in cat visual cortex. Proc Natl Acad Sci USA 92: 290-294, 1995.

LEE KH, WILLIAMS LM, BREAKSPEAR M, GORDON E: Synchronous gamma activity: a review and contribution to an integrative neuroscience model of schizophrenia. Brain Res Rev 41: 57-78, 2003.

LUTZENBERGER W, PULVERMULLER F, ELBERT T, BIRBAUMER N: Visual stimulation alters local 40-Hz responses in humans: an EEG study. Neurosci Lett 183: 39-42, 1995.

MEADOR KJ, RAY PG, ECHAUZ JR, LORING DW, VACHTSEVANOS GJ: Gamma coherence and conscious perception. Neurology 59: 1847-1854, 2005.

MENON V, FREEMAN WJ, CUTILLO BA, DESMOND JE, WARD MF, BRESSLER SL, LAXER KD, BARBARO N, GEVINS AS: Spatio-temporal correlations in human gamma band electrocorticograms. Electroencephalogr Clin Neurophysiol 98: 89-102, 1996.

MULLER MM, JUNGHOFER M, ELBERT T, ROCHSTROH B: Visually induced gamma-band responses to coherent and incoherent motion: a replication study. Neuroreport 8: 2575-2579, 1997.

OHARA S, MIMA T, BABA K, IKEDA A, KUNIEDA T, MATSUMOTO R, YAMAMOTO J, MATSUHASHI M, NAGAMINE T, HIRASAWA K, HORI T, MIHARA T, HASHIMOTO N, SALENIUS S, SHIBASAKI H: Increased synchronization of cortical oscillatory activities between human supplementary motor and primary sensorimotor areas during voluntary movements. $J$ Neurosci 21: 9377-9386, 2001.

PULVERMULLER F: Hebb's concept of cell assemblies and the psychophysiology of word processing. Psychophysiology 33: 317-333, 1996.

RODRIGUEZ R, GEORGE N, LACHAUX JP, MARTINERIE J, RENAULT B, VARELA FJ: Perception's shadow: long distance synchronization of human brain activity. Nature 397: 430-433, 1999.

RODRIGUEZ R, KALLENBACH U, SINGER W, MUNK MH: Short- and long-term effects of cholinergic modulation on gamma oscillations and response synchronization in the visual cortex. $J$ Neurosci 24: 10369-10378, 2004.

SCHOFFELEN JM, OOSTEVELD R, FRIES P: Neuronal coherence as a mechanism of effective corticospinal interaction. Science 308: 111-113, 2005.

TALAIRACH J, SZIKLA G, TOURNOUX P, PROSALENTIS A, BORDAS-FERRER M, COVELLO J: Atlas d'Anatomie Stereotaxique du Telencephale. Masson, Paris, 1967.

TALLON-BAUDRY C, BERTRAND O: Oscillatory activity in humans and its role in object representation. Trends Cogn Sci 3: 151-162, 1999. 